\title{
A NOTE ON STABLE HOMOTOPY MODULES
}

\author{
DONALD W. KAHN ${ }^{1}$ \\ ABstract. We establish under very general conditions that a \\ space may be changed by adding one cell, so that the stable \\ homotopy module becomes infinitely generated over the stable \\ homotopy ring. We give an application to the study of ideals in the \\ stable homotopy ring.
}

The stable homotopy groups of a finite $\mathrm{CW}$-complex form a graded module over the stable homotopy ring $G_{*}$. There has been some interest in these modules, largely due to the "Generating Hypothesis" of [2], which asserts that the functor which associates, with each finite complex, its stable homotopy module, is an imbedding in the category-theoretic sense. Although we do not know if this hypothesis is true, we now know that it has the following statements as corollaries.

(1) If $X$ is a finite complex, the stable homotopy module of $X$, $\pi_{*}^{S}(X)$, is finitely-generated over $G_{*}$, when and only when $X$ has the stable homotopy-type of a bouquet of spheres.

(2) $G_{*}$ is totally noncoherent in the sense that no nontrivial, finitely-generated, ideal of the ring $G_{*}$ is finitely presented.

In spite of our ignorance about the generating hypothesis, I established in earlier papers, [3] and [4], that $\pi_{*}^{S}(X)$ is of ten infinitely-generated. In fact, it is shown in [4], that if a finite complex $X$ has a nonzero rational homology group in some positive dimension, then we may add one cell to some suspension of $X$, and thus obtain a complex with infinitely-generated stable homotopy module. The purpose of the present paper is to prove the following stronger theorem.

THEOREM 1. Let $X$ be a finite complex. If there is an odd prime $p$ such that $H_{n}\left(X, Z_{p}\right) \neq 0$ for some $n>0$, then we may find $i>0$ and $\alpha: S^{m-1}$ $\rightarrow \Sigma^{i} X$ so that $\left(\Sigma^{i} X\right) \cup_{\alpha} e^{m}$ has infinitely-generated stable homotopy module.

In a sense, excluding 2-torsion, statement (1) above is within one cell of being true.

Now, one may easily see that statement (2) actually follows from statement (1). We shall thus also obtain

Received by the editors October 2, 1969.

AMS 1969 subject classifications. Primary 5540; Secondary 5530.

Key words and phrases. Stable homotopy, module, coherent.

1 Partially supported by contract NSF GP-5252. 
Theorem 2. Any nonzero, finitely-generated, ideal of $G_{*}$, which contains no element of infinite order, but contains some element of odd order, must be infinitely-presented. That is, if $\alpha$ is a map

$$
S^{n_{1}} \vee \cdots \vee S^{n_{k}} \stackrel{\alpha}{\rightarrow} S^{m}, \quad 2 m-1>n_{i}, 1 \leqq i \leqq k, n_{i}>m,
$$

whose image, on stable homotopy, is our ideal, then $\operatorname{ker}\left(\alpha_{*}\right)$ is infinitelygenerated.

We now pass to the proofs. I will use the stable homotopy exact couple [5] of a finite complex $X$, which has an associated spectral sequence whose $E^{1}$-term is a free, bigraded module on generators in $E_{n, 0}^{1}$, one for each $n$-cell of $X$. The spectral sequence converges to $\pi_{*}^{S}(X)$. It is shown in [4], that if $\operatorname{dim}(X)=K$ and $E_{K, *}^{\infty}$ is infinitelygenerated over $G_{*}$, then so is $\pi_{*}^{S}(X)$. Consider $X$ with some $H_{n}\left(X, Z_{p}\right)$ $\neq 0$. According to $[1], \pi_{i}^{\mathcal{S}}(X)$ has elements in $p$-torsion for infinitelymany $i$. Consequently, in the spectral sequence for $X, E_{r, *}^{\infty}(X)=E_{r, *}^{\infty}$ must have nonzero $p$-torsion for infinitely-many values $*$, for at least one $r$. Select the smallest such $r$ and ignore, from now on, torsion prime to $p$.

By our conventions, if $r_{1}<r, E_{r_{1}}^{\infty} *(X)$ is nonzero only for finitelymany values of $*$. We may then choose $m>\operatorname{dim}(X)$, so that if $r_{1}<r$, $E_{\tau_{1}, m_{1}}^{\infty}(X)=0$, when $m_{1} \geqq m$.

Choose $s>m$ so that $E_{r, s}^{\infty}(X)$ is nonzero, and let $u \in E_{r, s}^{\infty}(X)$ generate a summand of $p$-torsion. We let $u^{\prime} \in \pi_{r+s}^{s}\left(X^{r}\right)$ represent $u$, and $u^{\prime \prime}$ be the image of $u^{\prime}$ in $\pi_{r+s}^{s}(X)$. After suitable suspension, we form $X^{\prime}=\left(\sum i X\right) \cup_{u^{\prime \prime}} e$; clearly, $\operatorname{dim}\left(X^{\prime}\right)=r+s+i+1$, because we have chosen $s$ large. We note that $E_{*, *}^{l}(X)=E_{*, *}^{\infty}(X)$, whenever $l$ $>\operatorname{dim}(X)$, and that there is a natural map of the spectral sequence of $\Sigma i X$ to that of $X^{\prime}$.

We let $i_{e} \in E_{r+8+i+1,0}^{1}\left(X^{\prime}\right)$ be the generator corresponding to the cell $e$. It is easy to see that the first nonzero differential on $\left\{i_{e}\right\}$ is $d^{s+1}$, which maps $\left\{i_{e}\right\}$ to the class represented by $u^{\prime}$. Because of our assumptions on $r$, the range of all the higher differentials is null. Hence, we have shown that

$$
E_{r+s+i+1, *}^{\infty}\left(X^{\prime}\right)=\operatorname{ker}\left(d^{s+1}\right) .
$$

The proof will be completed by showing that this kernel is infinitelygenerated over $G_{*}$.

In the lowest dimension, $\operatorname{ker}\left(d^{s+1}\right)$ is infinite-cyclic, generated by $p^{t} i_{e}$, for some $t \geqq 1$ which is determined by the order of $u$. If we suppose our kernel is finitely-generated by $\gamma_{1}, \cdots, \gamma_{d}, \operatorname{dim} \gamma_{j}>\operatorname{dim} \gamma_{1}$, 
for $j>1$, then it is no loss of generality to take $\gamma_{1}=\left\{p^{i} i_{e}\right\}$. We shall also use $u$ to denote an element in $E_{r, s}^{1}(X)$ which passes to $u$ in $E_{r, s}^{\infty}(X)$; since $E_{r, *}^{1}(X)$ is free over $G_{*}, u$ may also be considered as a linear combination of elements in $G_{*} \oplus \cdots \oplus G_{*}$. We let $M$ be such that $h \cdot p^{M} \gamma_{j}=0, j>1$, with $(h, p)=1$.

According to [6] and [0], we may find an element $\alpha \in G_{*}$ such that

(1) $\alpha \in \operatorname{Im}(J)$.

(2) $p^{N} \alpha \neq 0, p^{N+1} \alpha=0$, for some $N>M$.

(3) $\alpha$ generates a summand.

(4) $\alpha \cdot u=0$.

(5) $\operatorname{dim}(\alpha)$ is as large as desired.

(1) and (2) follow from [6], while [0] allows us to have (3). (1) and (5) imply (4), because the domain of $J$ contains no $p$-torsion.

Because $\alpha \cdot u=0,\left\{\alpha \cdot i_{e}\right\} \in$ ker $d^{s+1}$. Thus, we may write $\left\{\alpha i_{e}\right\}$ $=\sum_{i} c_{i} \gamma_{i} ;$ multiplying both sides by $p^{N}$, we are left with

$$
0 \neq p^{N}\left\{\alpha \cdot i_{e}\right\}=p^{N} c_{1} \gamma_{1}=p^{N+t}\left\{c_{1} \cdot i_{e}\right\}
$$

Because $\alpha \cdot i_{e}$ generates a summand of order $p^{N+1}, c_{1} \cdot i_{e}$ cannot possibly have a component in this summand which is not annihilated by multiplication with $p^{N+t}, t \geqq 1$. Thus, our last equation is absurd, and $\operatorname{ker}\left(d^{s+1}\right)$ must be infinitely-generated. This completes the proof of Theorem 1.

Before proving Theorem 2, we prove the following:

Proposition. Let $\alpha_{i}: S^{n_{i}} \rightarrow S^{m}, n_{i} \ll 2 m-1, n_{i}>m, 1 \leqq i \leqq k$. Suppose that some $\alpha_{i}$ has order $h \cdot 2^{t}$, with $(h, 2)=1, h>1$. Then,

$$
X=S^{m} \cup \alpha_{i^{v}} \ldots v \alpha_{k}\left(e^{n_{1}+1} \cup \ldots \cup e^{n_{k}+1}\right)
$$

has infinitely-generated stable homotopy module.

ProOF. Let $p$ be a prime dividing $h$, and ignore torsion prime to $p$. Let $\left\{\alpha_{j}\right\}$ be the element of $G_{*} \approx \pi_{*}^{S}\left(S^{m}\right)$, of maximum dimension, whose order is not prime to $p$, among the attaching maps of the complex $X$.

As in the previous proof, it suffices to show that $\operatorname{ker}\left(d^{n_{j}+1-m}\right)$ is infinitely-generated over $G_{*}$. Letting $\gamma_{1}=\left(p^{a}\right) i, \gamma_{2}, \cdots, \gamma_{k}$ be the generators, we know that $\gamma_{2}, \cdots, \gamma_{k}$ are all of finite order. We select an element $\alpha$ as above, and use the identical argument to show that if $\gamma_{1}, \cdots, \gamma_{k}$ were in fact generators, then we must have a contradiction to the fact that $\alpha$ generates a summand. This proposition is similar to Theorem 1, except that we do not need to add any cells to our given complex. 
We now indicate the proof of Theorem 2. Consider a map

$$
S^{n_{1}} \vee \cdots \vee S^{n_{k}} \stackrel{\alpha}{\rightarrow} S^{m}
$$

as given. By the hypothesis, $n_{i}>m$ for all $i$, and the restriction of $\alpha$ to some summand is a map of finite order, which is not purely a power of 2. The cone on $\alpha$ is a space which satisfies the hypothesis of the above proposition. Considering the stable homotopy exact sequence

$$
\cdots \rightarrow \underset{\pi_{*}}{S}\left(S^{n_{1}} \vee \cdots \vee S^{n_{k}}\right) \stackrel{\alpha_{t}}{\longrightarrow} \underset{\pi_{*}}{S}\left(S^{m}\right) \stackrel{P_{t}}{\longrightarrow} \pi_{*}^{S}(C \alpha) \stackrel{\partial_{t}}{\longrightarrow} \cdots
$$

We extract a short exact sequence in the category of graded $G_{*-}$ modules:

$$
0 \rightarrow \operatorname{Im}\left(P_{\ddagger}\right) \rightarrow \pi_{*}^{\boldsymbol{s}}(C \alpha) \rightarrow \operatorname{ker}\left(\alpha_{\sharp}\right) \rightarrow 0 .
$$

Because $\operatorname{Im}\left(P_{\sharp}\right)$ is finitely-generated, we see that if $\operatorname{ker}(\alpha \ddagger)$ were finitely-generated, then $\pi_{*}^{\mathcal{S}}(C \alpha)$ would also be finitely-generated, contradicting the above proposition. This completes the proof of Theorem 2 .

\section{BIBLIOGRAPHY}

0. J. F. Adams, On the groups $J(X)$.IV, Topology 5 (1966), 21-71. MR 33 \#6628.

1. J. Cohen, Coherent graded rings and the non-existence of spaces of finite stable homotopy-type, Comment. Math. Helv. 44 (1969), 217-228.

2. P. Freyd, Stable homotopy, Proc. Conference Categorical Algebra (La Jolla, Calif., 1965), Springer-Verlag, New York, 1966, pp. 121-172. MR 35 \#2280.

3. D. Kahn, On stable homotopy modules, Invent. Math. 1 (1966), 375-379. MR 34 \#2015.

4. - On stable homotopy modules. II, Invent. Math. 7 (1969), 344-353. MR 39 \#7597.

5. - The homotopy category of exact couples, Topology 7 (1968), 233-253. MR $38 \# 1673$.

6. J. Milnor and M. Kervaire, Bernoulli numbers, homotopy groups, and a theorem of Rohlin, Proc. Internat. Congress Math. (Edinburgh, 1958), Cambridge Univ. Press, New York, 1960. MR 22 \#12531.

University of Minnesota, Minneapolis, Minnesota 55455 